University of Nebraska - Lincoln

DigitalCommons@University of Nebraska - Lincoln

$1-28-2002$

\title{
Measurement of the Strong Coupling Constant from Inclusive Jet Production at the Tevatron $\bar{p} p$ Collider
}

\author{
T. Affolder \\ Ernest Orlando Lawrence Berkeley National Laboratory, Berkeley, California \\ Kenneth A. Bloom \\ University of Nebraska-Lincoln, kenbloom@unl.edu \\ Collider Detector at Fermilab Collaboration
}

Follow this and additional works at: https://digitalcommons.unl.edu/physicsbloom

Part of the Physics Commons

Affolder, T.; Bloom, Kenneth A.; and Fermilab Collaboration, Collider Detector at, "Measurement of the Strong Coupling Constant from Inclusive Jet Production at the Tevatron $\bar{p} p$ Collider" (2002). Kenneth Bloom Publications. 71.

https://digitalcommons.unl.edu/physicsbloom/71

This Article is brought to you for free and open access by the Research Papers in Physics and Astronomy at DigitalCommons@University of Nebraska - Lincoln. It has been accepted for inclusion in Kenneth Bloom Publications by an authorized administrator of DigitalCommons@University of Nebraska - Lincoln. 


\section{Measurement of the Strong Coupling Constant from Inclusive Jet Production at the Tevatron $\bar{p} p$ Collider}

T. Affolder, ${ }^{1}$ H. Akimoto, ${ }^{2}$ A. Akopian, ${ }^{3}$ M. G. Albrow ${ }^{4}$ P. Amaral,${ }^{5}$ D. Amidei, ${ }^{6}$ K. Anikeev, ${ }^{7}$ J. Antos, ${ }^{8}$ G. Apollinari, ${ }^{4}$ T. Arisawa, ${ }^{2}$ A. Artikov, ${ }^{9}$ T. Asakawa, ${ }^{10}$ W. Ashmanskas,${ }^{5}$ F. Azfar, ${ }^{11}$ P. Azzi-Bacchetta, ${ }^{12}$ N. Bacchetta, ${ }^{12}$ H. Bachacou, ${ }^{1}$ S. Bailey,${ }^{13}$ P. de Barbaro, ${ }^{14}$ A. Barbaro-Galtieri, ${ }^{1}$ V. E. Barnes, ${ }^{15}$ B. A. Barnett, ${ }^{16}$ S. Baroiant, ${ }^{17}$ M. Barone, ${ }^{18}$ G. Bauer ${ }^{19}$ F. Bedeschi, ${ }^{20}$ S. Belforte, ${ }^{21}$ W. H. Bell, ${ }^{22}$ G. Bellettini, ${ }^{20}$ J. Bellinger, ${ }^{23}$ D. Benjamin, ${ }^{24}$ J. Bensinger, ${ }^{25}$ A. Beretvas, ${ }^{4}$ J.P. Berge, ${ }^{4}$ J. Berryhill,${ }^{5}$ A. Bhatti, ${ }^{3}$ M. Binkley, ${ }^{4}$ D. Bisello, ${ }^{12}$ M. Bishai, ${ }^{4}$ R.E. Blair ${ }^{26}$ C. Blocker, ${ }^{25}$ K. Bloom, ${ }^{6}$ B. Blumenfeld, ${ }^{16}$ S. R. Blusk, ${ }^{14}$ A. Bocci, ${ }^{3}$ A. Bodek,${ }^{14}$ W. Bokhari, ${ }^{27}$ G. Bolla, ${ }^{15}$ Y. Bonushkin, ${ }^{28}$ D. Bortoletto, ${ }^{15}$ J. Boudreau, ${ }^{29}$ A. Brandl, ${ }^{30}$ S. van den Brink, ${ }^{16}$ C. Bromberg, ${ }^{31}$ M. Brozovic, ${ }^{24}$ E. Brubaker, ${ }^{1}$ N. Bruner, ${ }^{30}$ E. Buckley-Geer, ${ }^{4}$ J. Budagov, ${ }^{9}$ H. S. Budd, ${ }^{14}$ K. Burkett, ${ }^{13}$ G. Busetto, ${ }^{12}$ A. Byon-Wagner, ${ }^{4}$ K. L. Byrum, ${ }^{26}$ S. Cabrera,${ }^{24}$ P. Calafiura, ${ }^{1}$ M. Campbell, ${ }^{6}$ W. Carithers, ${ }^{1}$ J. Carlson, ${ }^{6}$ D. Carlsmith, ${ }^{23}$ W. Caskey, ${ }^{17}$ A. Castro, ${ }^{32}$ D. Cauz,${ }^{21}$ A. Cerri, ${ }^{20}$ A. W. Chan,${ }^{8}$ P. S. Chang, ${ }^{8}$ P. T. Chang, ${ }^{8}$ J. Chapman, ${ }^{6}$ C. Chen, ${ }^{27}$ Y. C. Chen, ${ }^{8}$ M.-T. Cheng, ${ }^{8}$ M. Chertok, ${ }^{17}$ G. Chiarelli, ${ }^{20}$ I. Chirikov-Zorin, ${ }^{9}$ G. Chlachidze, ${ }^{9}$ F. Chlebana, ${ }^{4}$ L. Christofek, ${ }^{33}$ M.L. Chu, ${ }^{8}$ Y. S. Chung, ${ }^{14}$ C. I. Ciobanu, ${ }^{34}$ A. G. Clark, ${ }^{35}$ A. P. Colijn, ${ }^{4}$ A. Connolly,,${ }^{1}$ M. Convery, ${ }^{3}$ J. Conway, ${ }^{36}$ M. Cordelli, ${ }^{18}$ J. Cranshaw, ${ }^{37}$ R. Cropp,${ }^{38}$ R. Culbertson, ${ }^{4}$ D. Dagenhart, ${ }^{39}$ S. D' Auria, ${ }^{22}$ F. DeJongh, ${ }^{4}$ S. Dell'Agnello, ${ }^{18}$ M. Dell'Orso, ${ }^{20}$ S. Demers, ${ }^{3}$ L. Demortier, ${ }^{3}$ M. Deninno, ${ }^{32}$ P. F. Derwent, ${ }^{4}$ T. Devlin, ${ }^{36}$ J. R. Dittmann, ${ }^{4}$ A. Dominguez, ${ }^{1}$ S. Donati, ${ }^{20}$ J. Done,,${ }^{40}$ M. D'Onofrio,${ }^{20}$ T. Dorigo, ${ }^{13}$ N. Eddy,${ }^{33}$ K. Einsweiler, ${ }^{1}$ J.E. Elias, ${ }^{4}$ E. Engels, Jr. ${ }^{29}$ R. Erbacher, ${ }^{4}$ D. Errede, ${ }^{33}$ S. Errede, ${ }^{33}$ Q. Fan, ${ }^{14}$ H.-C. Fang, ${ }^{1}$ R. G. Feild, ${ }^{41}$ J. P. Fernandez,${ }^{4}$ C. Ferretti, ${ }^{20}$ R. D. Field, ${ }^{42}$ I. Fiori, ${ }^{32}$ B. Flaugher, ${ }^{4}$ G. W. Foster,${ }^{4}$ M. Franklin, ${ }^{13}$ J. Freeman, ${ }^{4}$ J. Friedman ${ }^{19}$ Y. Fukui, ${ }^{43}$ I. Furic, ${ }^{19}$ S. Galeotti, ${ }^{20}$ A. Gallas, ${ }^{13}, *$ M. Gallinaro, ${ }^{3}$ T. Gao, ${ }^{27}$ M. Garcia-Sciveres, ${ }^{1}$ A. F. Garfinkel, ${ }^{15}$ P. Gatti, ${ }^{12}$ C. Gay, ${ }^{41}$ D. W. Gerdes, ${ }^{6}$ P. Giannetti, ${ }^{20}$ P. Giromini, ${ }^{18}$ V. Glagolev, ${ }^{9}$ D. Glenzinski, ${ }^{4}$ M. Gold,${ }^{30}$ J. Goldstein, ${ }^{4}$ I. Gorelov, ${ }^{30}$ A. T. Goshaw, ${ }^{24}$ Y. Gotra, ${ }^{29}$ K. Goulianos, ${ }^{3}$ C. Green, ${ }^{15}$ G. Grim, ${ }^{17}$ P. Gris,${ }^{4}$ L. Groer,${ }^{36}$ C. Grosso-Pilcher, ${ }^{5}$ M. Guenther, ${ }^{15}$ G. Guillian, ${ }^{6}$ J. Guimaraes da Costa, ${ }^{13}$ R. M. Haas, ${ }^{42}$ C. Haber, ${ }^{1}$ S. R. Hahn, ${ }^{4}$ C. Hall, ${ }^{13}$ T. Handa, ${ }^{44}$ R. Handler, ${ }^{23}$ W. Hao ${ }^{37}$ F. Happacher,${ }^{18}$ K. Hara, ${ }^{10}$ A. D. Hardman, ${ }^{15}$ R. M. Harris,${ }^{4}$ F. Hartmann, ${ }^{45}$ K. Hatakeyama, ${ }^{3}$ J. Hauser ${ }^{28}$ J. Heinrich, ${ }^{27}$ A. Heiss, ${ }^{45}$ M. Herndon, ${ }^{16}$ C. Hill, ${ }^{17}$ K. D. Hoffman, ${ }^{15}$ C. Holck, ${ }^{27}$ R. Hollebeek, ${ }^{27}$

L. Holloway, ${ }^{33}$ B. T. Huffman, ${ }^{11}$ R. Hughes, ${ }^{34}$ J. Huston, ${ }^{31}$ J. Huth, ${ }^{13}$ H. Ikeda, ${ }^{10}$ J. Incandela,,${ }^{4,}$ G. Introzzi, ${ }^{20}$ A. Ivanov, ${ }^{14}$ J. Iwai, ${ }^{2}$ Y. Iwata, ${ }^{44}$ E. James, ${ }^{6}$ M. Jones, ${ }^{27}$ U. Joshi, ${ }^{4}$ H. Kambara, ${ }^{35}$ T. Kamon, ${ }^{40}$ T. Kaneko, ${ }^{10}$ K. Karr, ${ }^{39}$ S. Kartal, ${ }^{4}$ H. Kasha, ${ }^{41}$ Y. Kato, ${ }^{46}$ T. A. Keaffaber,${ }^{15}$ K. Kelley, ${ }^{19}$ M. Kelly, ${ }^{6}$ D. Khazins, ${ }^{24}$ T. Kikuchi, ${ }^{10}$ B. Kilminster, ${ }^{14}$ B. J. Kim, ${ }^{47}$ D. H. Kim,${ }^{47}$ H. S. Kim,${ }^{33}$ M. J. Kim,${ }^{47}$ S. B. Kim, ${ }^{47}$ S. H. Kim, ${ }^{10}$ Y. K. Kim, ${ }^{1}$ M. Kirby, ${ }^{24}$ M. Kirk, ${ }^{25}$ L. Kirsch, ${ }^{25}$ S. Klimenko, ${ }^{42}$ P. Koehn, ${ }^{34}$ K. Kondo, ${ }^{2}$ J. Konigsberg, ${ }^{42}$ A. Korn, ${ }^{19}$ A. Korytov, ${ }^{42}$ E. Kovacs, ${ }^{26}$ J. Kroll, ${ }^{27}$ M. Kruse, ${ }^{24}$ S. E. Kuhlmann, ${ }^{26}$ K. Kurino, ${ }^{44}$ T. Kuwabara, ${ }^{10}$ A. T. Laasanen, ${ }^{15}$ N. Lai, ${ }^{5}$ S. Lami, ${ }^{3}$ S. Lammel, ${ }^{4}$ J. Lancaster, ${ }^{24}$ M. Lancaster, ${ }^{1}$ R. Lander, ${ }^{17}$ A. Lath, ${ }^{36}$ G. Latino, ${ }^{20}$ T. LeCompte, ${ }^{26}$ A. M. Lee IV,${ }^{24}$ K. Lee, ${ }^{37}$ S. Leone, ${ }^{20}$ J. D. Lewis, ${ }^{4}$ M. Lindgren, ${ }^{28}$ T. M. Liss, ${ }^{33}$ J. B. Liu, ${ }^{14}$ Y.C. Liu, ${ }^{8}$ D. O. Litvintsev, ${ }^{4}$ O. Lobban, ${ }^{37}$ N. Lockyer, ${ }^{27}$ J. Loken, ${ }^{11}$ M. Loreti, ${ }^{12}$ D. Lucchesi, ${ }^{12}$ P. Lukens, ${ }^{4}$ S. Lusin, ${ }^{23}$ L. Lyons, ${ }^{11}$ J. Lys, ${ }^{1}$ R. Madrak, ${ }^{13} \mathrm{~K}$. Maeshima, ${ }^{4}$ P. Maksimovic, ${ }^{13}$ L. Malferrari, ${ }^{32}$ M. Mangano, ${ }^{20}$ M. Mariotti, ${ }^{12}$ G. Martignon, ${ }^{12}$ A. Martin, ${ }^{41}$ J. A. J. Matthews,${ }^{30}$ J. Mayer, ${ }^{38}$ P. Mazzanti, ${ }^{32}$ K. S. McFarland, ${ }^{14}$ P. McIntyre, ${ }^{40}$ E. McKigney, ${ }^{27}$ M. Menguzzato, ${ }^{12}$ A. Menzione,${ }^{20}$ P. Merkel, ${ }^{4}$ C. Mesropian, ${ }^{3}$ A. Meyer, ${ }^{4}$ T. Miao, ${ }^{4}$ R. Miller, ${ }^{31}$ J. S. Miller, ${ }^{6}$ H. Minato, ${ }^{10}$ S. Miscetti ${ }^{18}$ M. Mishina, ${ }^{43}$ G. Mitselmakher, ${ }^{42}$ Y. Miyazaki, ${ }^{46}$ N. Moggi, ${ }^{32}$ E. Moore,${ }^{30}$ R. Moore, ${ }^{6}$ Y. Morita, ${ }^{43}$ T. Moulik, ${ }^{15}$ M. Mulhearn, ${ }^{19}$ A. Mukherjee, ${ }^{4}$ T. Muller, ${ }^{45}$ A. Munar, ${ }^{20}$ P. Murat ${ }^{4}$ S. Murgia, ${ }^{31}$ J. Nachtman, ${ }^{28}$ V. Nagaslaev ${ }^{37}$ S. Nahn, ${ }^{41}$ H. Nakada ${ }^{10}$ I. Nakano, ${ }^{44}$ C. Nelson, ${ }^{4}$ T. Nelson, ${ }^{4}$ C. Neu, ${ }^{34}$ D. Neuberger,${ }^{45}$ C. Newman-Holmes, ${ }^{4}$ C.-Y.P. Ngan, ${ }^{19}$ H. Niu, ${ }^{25}$ L. Nodulman, ${ }^{26}$ A. Nomerotski, ${ }^{42}$ S. H. Oh ${ }^{24}$ Y. D. Oh, ${ }^{47}$ T. Ohmoto, ${ }^{44}$ T. Ohsugi, ${ }^{44}$ R. Oishi, ${ }^{10}$ T. Okusawa, ${ }^{46}$ J. Olsen, ${ }^{23}$ W. Orejudos, ${ }^{1}$ C. Pagliarone, ${ }^{20}$ F. Palmonari, ${ }^{20}$ R. Paoletti, ${ }^{20}$ V. Papadimitriou, ${ }^{37}$ D. Partos,${ }^{25}$ J. Patrick, ${ }^{4}$ G. Pauletta, ${ }^{21}$ M. Paulini, ${ }^{1,}$ C. Paus, ${ }^{19}$ D. Pellett, ${ }^{17}$ L. Pescara, ${ }^{12}$ T. J. Phillips, ${ }^{24}$ G. Piacentino, ${ }^{20}$ K. T. Pitts, ${ }^{33}$ A. Pompos,${ }^{15}$ L. Pondrom, ${ }^{23}$ G. Pope,${ }^{29}$ M. Popovic,${ }^{38}$ F. Prokoshin, ${ }^{9}$ J. Proudfoot, ${ }^{26}$ F. Ptohos,,${ }^{18}$ O. Pukhov, ${ }^{9}$ G. Punzi, ${ }^{20}$ A. Rakitine, ${ }^{19}$ F. Ratnikov, ${ }^{36}$ D. Reher,${ }^{1}$ A. Reichold,,${ }^{11}$ P. Renton, ${ }^{11}$ A. Ribon, ${ }^{12}$ W. Riegler,${ }^{13}$ F. Rimondi, ${ }^{32}$ L. Ristori, ${ }^{20}$ M. Riveline, ${ }^{38}$ W. J. Robertson, ${ }^{24}$ A. Robinson, ${ }^{38}$ T. Rodrigo, ${ }^{48}$ S. Rolli, ${ }^{39}$ L. Rosenson, ${ }^{19}$ R. Roser, ${ }^{4}$ R. Rossin, ${ }^{12}$ C. Rott, ${ }^{15}$ A. Roy, ${ }^{15}$ A. Ruiz, ${ }^{48}$ A. Safonov,${ }^{17}$ R. St. Denis, ${ }^{22}$ W. K. Sakumoto, ${ }^{14}$ D. Saltzberg, ${ }^{28}$ C. Sanchez, ${ }^{34}$ A. Sansoni, ${ }^{18}$ L. Santi, ${ }^{21}$ H. Sato, ${ }^{10}$ P. Savard ${ }^{38}$ P. Schlabach, ${ }^{4}$ E. E. Schmidt, ${ }^{4}$ M. P. Schmidt, ${ }^{41}$ M. Schmitt, ${ }^{13}, *$ L. Scodellaro, ${ }^{12}$ A. Scott ${ }^{28}$ A. Scribano, ${ }^{20}$ S. Segler, ${ }^{4}$ S. Seidel,${ }^{30}$ Y. Seiya,${ }^{10}$ A. Semenov, ${ }^{9}$ 
F. Semeria, ${ }^{32}$ T. Shah, ${ }^{19}$ M. D. Shapiro, ${ }^{1}$ P. F. Shepard, ${ }^{29}$ T. Shibayama, ${ }^{10}$ M. Shimojima, ${ }^{10}$ M. Shochet, ${ }^{5}$ A. Sidoti, ${ }^{12}$ J. Siegrist, ${ }^{1}$ A. Sill, ${ }^{37}$ P. Sinervo, ${ }^{38}$ P. Singh, ${ }^{33}$ A. J. Slaughter, ${ }^{41}$ K. Sliwa, ${ }^{39}$ C. Smith,${ }^{16}$ F. D. Snider, ${ }^{4}$ A. Solodsky, ${ }^{3}$ J. Spalding, ${ }^{4}$ T. Speer, ${ }^{35}$ P. Sphicas, ${ }^{19}$ F. Spinella, ${ }^{20}$ M. Spiropulu,${ }^{5}$ L. Spiegel, ${ }^{4}$ J. Steele, ${ }^{23}$ A. Stefanini, ${ }^{20}$ J. Strologas ${ }^{33}$ F. Strumia, ${ }^{35}$ D. Stuart, ${ }^{4}$ K. Sumorok, ${ }^{19}$ T. Suzuki, ${ }^{10}$ T. Takano,${ }^{46}$ R. Takashima,${ }^{44}$ K. Takikawa,${ }^{10}$ P. Tamburello, ${ }^{24}$ M. Tanaka, ${ }^{10}$ B. Tannenbaum, ${ }^{28}$ M. Tecchio, ${ }^{6}$ R. Tesarek,${ }^{4}$ P. K. Teng,${ }^{8}$ K. Terashi,${ }^{3}$ S. Tether, ${ }^{19}$ A. S. Thompson, ${ }^{22}$ R. Thurman-Keup, ${ }^{26}$ P. Tipton, ${ }^{14} \mathrm{~S}$. Tkaczyk, ${ }^{4} \mathrm{D}$. Toback, ${ }^{40} \mathrm{~K}$. Tollefson,,${ }^{14} \mathrm{~A}$. Tollestrup, ${ }^{4} \mathrm{D}$. Tonelli, ${ }^{20} \mathrm{H}$. Toyoda, ${ }^{46}$ W. Trischuk, ${ }^{38}$ J.F. de Troconiz, ${ }^{13}$ J. Tseng, ${ }^{19}$ N. Turini, ${ }^{20}$ F. Ukegawa, ${ }^{10}$ T. Vaiciulis, ${ }^{14} \mathrm{~J}$. Valls,${ }^{36} \mathrm{~S}$. Vejcik III, ${ }^{4}$ G. Velev, ${ }^{4}$ G. Veramendi, ${ }^{1}$ R. Vidal,${ }^{4}$ I. Vila ${ }^{48}$ R. Vilar, ${ }^{48}$ I. Volobouev, ${ }^{1}$ M. von der Mey,${ }^{28}$ D. Vucinic, ${ }^{19}$ R. G. Wagner, ${ }^{26}$ R. L. Wagner, ${ }^{4}$ N. B. Wallace, ${ }^{36}$ Z. Wan, ${ }^{36}$ C. Wang, ${ }^{24}$ M. J. Wang, ${ }^{8}$ B. Ward,${ }^{22}$ S. Waschke, ${ }^{22}$ T. Watanabe, ${ }^{10}$ D. Waters, ${ }^{11}$ T. Watts,,${ }^{36}$ R. Webb, ${ }^{40}$ H. Wenzel, ${ }^{45}$ W. C. Wester III, ${ }^{4}$ A. B. Wicklund, ${ }^{26}$ E. Wicklund, ${ }^{4}$ T. Wilkes, ${ }^{17}$ H. H. Williams, ${ }^{27}$ P. Wilson, ${ }^{4}$ B. L. Winer, ${ }^{34}$ D. Winn, ${ }^{6}$ S. Wolbers,${ }^{4}$ D. Wolinski, ${ }^{6}$ J. Wolinski, ${ }^{31}$ S. Wolinski, ${ }^{6}$ S. Worm, ${ }^{30}$ X. Wu, ${ }^{35}$ J. Wyss, ${ }^{20}$ W. Yao, ${ }^{1}$ G. P. Yeh, ${ }^{4}$ P. Yeh,${ }^{8}$ J. Yoh, ${ }^{4}$ C. Yosef, ${ }^{31}$ T. Yoshida, ${ }^{46} \mathrm{I} . ~ Y u,{ }^{47}$ S. Yu, ${ }^{27}$ Z. Yu, ${ }^{41}$ A. Zanetti, ${ }^{21}$ F. Zetti, ${ }^{1}$ and S. Zucchelli ${ }^{32}$

\title{
(CDF Collaboration)
}

\author{
${ }^{1}$ Ernest Orlando Lawrence Berkeley National Laboratory, Berkeley, California 94720 \\ ${ }^{2}$ Waseda University, Tokyo 169, Japan \\ ${ }^{3}$ Rockefeller University, New York, New York 10021 \\ ${ }^{4}$ Fermi National Accelerator Laboratory, Batavia, Illinois 60510 \\ ${ }^{5}$ Enrico Fermi Institute, University of Chicago, Chicago, Illinois 60637 \\ ${ }^{6}$ University of Michigan, Ann Arbor, Michigan 48109 \\ ${ }^{7}$ Massachusetts Institute of Technology, Cambridge, Massachusetts 02139 \\ ${ }^{8}$ Institute of Physics, Academia Sinica, Taipei, Taiwan 11529, Republic of China \\ ${ }^{9}$ Joint Institute for Nuclear Research, RU-141980 Dubna, Russia \\ ${ }^{10}$ University of Tsukuba, Tsukuba, Ibaraki 305, Japan \\ ${ }^{11}$ University of Oxford, Oxford OX1 3RH, United Kingdom \\ ${ }^{12}$ Universita di Padova, Istituto Nazionale di Fisica Nucleare, Sezione di Padova, I-35131 Padova, Italy \\ ${ }^{13}$ Harvard University, Cambridge, Massachusetts 02138 \\ ${ }^{14}$ University of Rochester, Rochester, New York 14627 \\ ${ }^{15}$ Purdue University, West Lafayette, Indiana 47907 \\ ${ }^{16}$ The Johns Hopkins University, Baltimore, Maryland 21218 \\ ${ }^{17}$ University of California at Davis, Davis, California 95616 \\ ${ }^{18}$ Laboratori Nazionali di Frascati, Istituto Nazionale di Fisica Nucleare, I-00044 Frascati, Italy \\ ${ }^{19}$ Massachusetts Institute of Technology, Cambridge, Massachusetts 02139 \\ ${ }^{20}$ Istituto Nazionale di Fisica Nucleare, University and Scuola Normale Superiore of Pisa, I-56100 Pisa, Italy \\ ${ }^{21}$ Istituto Nazionale di Fisica Nucleare, University of Trieste/Udine, Italy \\ ${ }^{22}$ Glasgow University, Glasgow G12 8QQ, United Kingdom \\ ${ }^{23}$ University of Wisconsin, Madison, Wisconsin 53706 \\ ${ }^{24}$ Duke University, Durham, North Carolina 27708 \\ ${ }^{25}$ Brandeis University, Waltham, Massachusetts 02254 \\ ${ }^{26}$ Argonne National Laboratory, Argonne, Illinois 60439 \\ ${ }^{27}$ University of Pennsylvania, Philadelphia, Pennsylvania 19104 \\ ${ }^{28}$ University of California at Los Angeles, Los Angeles, California 90024 \\ ${ }^{29}$ University of Pittsburgh, Pittsburgh, Pennsylvania 15260 \\ ${ }^{30}$ University of New Mexico, Albuquerque, New Mexico 87131 \\ ${ }^{31}$ Michigan State University, East Lansing, Michigan 48824 \\ ${ }^{32}$ Istituto Nazionale di Fisica Nucleare, University of Bologna, I-40127 Bologna, Italy \\ ${ }^{33}$ University of Illinois, Urbana, Illinois 61801 \\ ${ }^{34}$ The Ohio State University, Columbus, Ohio 43210 \\ ${ }^{35}$ University of Geneva, CH-1211 Geneva 4, Switzerland \\ ${ }^{36}$ Rutgers University, Piscataway, New Jersey 08855 \\ ${ }^{37}$ Texas Tech University, Lubbock, Texas 79409 \\ ${ }^{38}$ Institute of Particle Physics, University of Toronto, Toronto M5S 1A7, Canada \\ ${ }^{39}$ Tufts University, Medford, Massachusetts 02155 \\ ${ }^{40}$ Texas A\&M University, College Station, Texas 77843 \\ ${ }^{41}$ Yale University, New Haven, Connecticut 06520 \\ ${ }^{42}$ University of Florida, Gainesville, Florida 32611 \\ ${ }^{43}$ High Energy Accelerator Research Organization (KEK), Tsukuba, Ibaraki 305, Japan
}




\author{
${ }^{44}$ Hiroshima University, Higashi-Hiroshima 724, Japan \\ ${ }^{45}$ Institut für Experimentelle Kernphysik, Universität Karlsruhe, 76128 Karlsruhe, Germany \\ ${ }^{46}$ Osaka City University, Osaka 588, Japan \\ ${ }^{47}$ Center for High Energy Physics, Kyungpook National University, Taegu 702-701, Korea \\ and Seoul National University, Seoul 151-742, Korea \\ and SungKyunKwan University, Suwon 440-746, Korea \\ ${ }^{48}$ Instituto de Fisica de Cantabria, CSIC-University of Cantabria, 39005 Santander, Spain
}

(Received 29 August 2001; published 9 January 2002)

\begin{abstract}
We report a measurement of the strong coupling constant, $\alpha_{s}\left(M_{Z}\right)$, extracted from inclusive jet production in $p \bar{p}$ collisions at $\sqrt{s}=1800 \mathrm{GeV}$. The QCD prediction for the evolution of $\alpha_{s}$ with jet transverse energy $E_{T}$ is tested over the range $40<E_{T}<450 \mathrm{GeV}$ using $E_{T}$ for the renormalization scale. The data show good agreement with QCD in the region below $250 \mathrm{GeV}$. The value of $\alpha_{s}$ at the mass of the $Z^{0}$ boson averaged over the range $40<E_{T}<250 \mathrm{GeV}$ is found to be $\alpha_{s}\left(M_{Z}\right)=$ $0.1178 \pm 0.0001$ (stat) ${ }_{-0.0095}^{+0.0081}$ (expt. syst). The associated theoretical uncertainties are mainly due to the choice of renormalization scale $\left(\begin{array}{c}+6 \% \\ -4 \%\end{array}\right)$ and input parton distribution functions $(5 \%)$.
\end{abstract}

PACS numbers: 12.38.Qk, 13.87.Ce

Jet production at hadron colliders provides an excellent opportunity for testing the theory of strong interactions, quantum chromodynamics (QCD) [1]. QCD has achieved remarkable success in describing hadron interactions at short distances (large momentum transfers), owing to the property of asymptotic freedom [2]. Asymptotic freedom predicts a logarithmic decrease of the coupling strength, $\alpha_{s}(\mu)$, as the momentum scale $\mu$ characterizing a process increases. Processes with large momentum transfer can then be described by an expansion in powers of $\alpha_{s}(\mu)$. The value of $\alpha_{s}$, a free parameter of QCD, is one of the fundamental constants of nature. Its determination is the essential measurement of QCD, and the observation of its evolution, or running, with momentum transfer is one of the key tests of the theory. At $e^{+} e^{-}$colliders $\alpha_{s}$ has been measured from the fragmentation functions [3], event shapes [4], jet production rates [5], and $\tau$ lepton decay properties [6]. In lepton-hadron collisions, $\alpha_{s}$ has been measured from scaling violations [7], jet production rates [8], and momentum sum rules [9]. A precise value for $\alpha_{s}$ has also been obtained from a global fit to properties of the $Z^{0}$ boson measured at the CERN LEP and the SLAC SLC $e^{+} e^{-}$colliders and the $W$ boson and top quark masses [10]. A review of these and other $\alpha_{s}$ measurements can be found in [11]. In this Letter, we present a measurement of $\alpha_{s}$ from the inclusive jet cross section in $\bar{p} p$ collisions over the jet transverse energy $\left(E_{T}\right)$ range from 40 to $450 \mathrm{GeV}$.

This measurement is based on a data sample of integrated luminosity $87 \mathrm{pb}^{-1}$ collected by the Collider Detector at Fermilab (CDF) during the 1994-1995 run (Run 1b) of the Fermilab Tevatron $\bar{p} p$ collider operating at $\sqrt{s}=$ $1.8 \mathrm{TeV}$. The CDF detector is described elsewhere [12]. Details of the measurement of the inclusive jet differential cross section can be found in [13]. Briefly, jets are reconstructed using an iterative fixed cone algorithm with a radius $R=\left(\Delta \eta^{2}+\Delta \phi^{2}\right)^{1 / 2}=0.7$, where $\eta \equiv$ $-\ln \left(\tan \frac{\theta}{2}\right)$ is the pseudorapidity, evaluated from the angle $\theta$ between the centerline of the jet cone and the proton beam line, and $\phi$ is the azimuthal angle. The inclusive jet cross section includes all jets in an event in the pseudorapidity range $0.1<|\eta|<0.7$. The measured spectrum is corrected for the calorimeter response, resolution, and the underlying event energy using an iterative unsmearing procedure which changes both the energy scale and the normalization simultaneously. The value of $\alpha_{s}$ is determined by comparing the jet cross section with the next to leading order (NLO) perturbative QCD predictions [14]. In the $E_{T}$ region studied, the nonperturbative contributions to the inclusive jet cross section are estimated to be negligible [15]. The procedure of extracting $\alpha_{s}$ can be summarized by the equation

$$
\begin{aligned}
\frac{d \sigma}{d E_{T}}= & \alpha_{s}^{2}\left(\mu_{R}\right) \hat{X}^{(0)}\left(\mu_{F}, E_{T}\right) \\
& \times\left[1+\alpha_{s}\left(\mu_{R}\right) k_{1}\left(\mu_{R}, \mu_{F}, E_{T}\right)\right],
\end{aligned}
$$

where $\frac{d \sigma}{d E_{T}}$ is the transverse energy distribution of the inclusive jets, $\mu_{R}$ and $\mu_{F}$, related to $E_{T}$ by a scale factor, are the renormalization and factorization scales, $\alpha_{s}^{2}\left(\mu_{R}\right) \hat{X}^{(0)}\left(\mu_{F}, E_{T}\right)$ is the leading order (LO) prediction for the inclusive jet cross section, and $\alpha_{s}^{3}\left(\mu_{R}\right) \hat{X}^{(0)}\left(\mu_{F}, E_{T}\right) k_{1}\left(\mu_{R}, \mu_{F}, E_{T}\right)$ is the NLO contribution. Both $\hat{X}^{(0)}\left(\mu_{F}, E_{T}\right)$ and $k_{1}\left(\mu_{R}, \mu_{F}, E_{T}\right)$ are calculated with the JETRAD Monte Carlo program [16] based on the techniques described in [17] and the matrix elements of [18]. NLO QCD predictions for the inclusive jet cross section are also available in $[19,20]$ and agree well with those of JETRAD. All calculations are performed in the modified minimal subtraction, $\overline{\mathrm{MS}}$, scheme [21]. The JETRAD Monte Carlo program generates events with weighting factors, so that the jet clustering algorithm and $E_{T}$ and $\eta$ cuts, mimicking the experimental requirements, are directly applied to the final state partons. The jet clustering in JETRAD is governed by a cone radius $R$ and a phenomenological parameter $\mathcal{R}_{\text {sep }}$ (default value $=1.3$ ), introduced to match the experimental efficiency of identifying overlapping jets [15]. If two partons are more than 
$\mathcal{R}_{\text {sep }} \times R$ apart, they are identified as two distinct jets; otherwise they are merged into a single jet.

The inclusive jet data are divided into $33 E_{T}$ bins, from which we obtain statistically independent measurements of $\alpha_{s}$ for 33 different values of $\mu_{R}$. The $\alpha_{s}$ values derived for $\mu_{R}=\mu_{F}=E_{T}$ using CTEQ4M [22] parton distribution functions (PDFs) are presented in Fig. 1. For $E_{T}<250 \mathrm{GeV}$, there is good agreement with QCD predictions for the running of the coupling constant. The behavior of $\alpha_{s}$ at higher $E_{T}$ values is a direct reflection of the excess observed in the inclusive jet cross section [13]. The discrepancy with the NLO QCD predictions in this region, though not well understood, may be accommodated by the flexibility allowed by the world data in determining the high- $x$ gluon component in the parton distributions [22].

The measured values of $\alpha_{s}\left(\mu_{R}\right)$ are evolved to the mass of the $Z^{0}$ boson, $M_{Z}$, by using the solution to the two-loop renormalization group equation:

$$
\alpha_{s}\left(M_{Z}\right)=\frac{\alpha_{s}\left(\mu_{R}\right)}{1-\alpha_{s}\left(\mu_{R}\right)\left[b_{0}+b_{1} \alpha_{s}\left(\mu_{R}\right)\right] \ln \left(\mu_{R} / M_{Z}\right)},
$$

$$
b_{0}=\frac{33-2 n_{f}}{6 \pi} \quad b_{1}=\frac{306-38 n_{f}}{24 \pi^{2}},
$$

where $n_{f}$ is the number of active flavors, which is equal to five (six) for $\mu_{R}$ smaller (larger) than the top quark mass. The values of $\alpha_{s}\left(M_{Z}\right)$ for all 33 measurements are

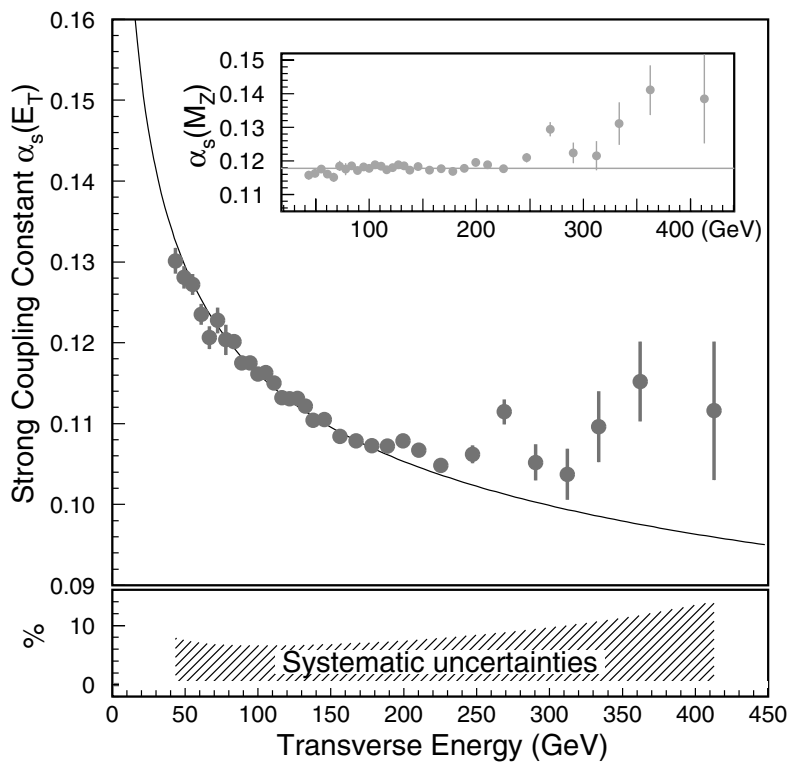

FIG. 1. The strong coupling constant as a function of $E_{T}$ for $\mu_{R}=E_{T}$ measured using CTEQ4M parton distributions. The shaded area shows the experimental systematic uncertainties. The curved line represents the NLO QCD prediction for the evolution of $\alpha_{s}\left(E_{T}\right)$ using $\alpha_{s}\left(M_{Z}\right)=0.1178$, the average value obtained in the region $40<E_{T}<250 \mathrm{GeV}$. The $\alpha_{s}\left(M_{Z}\right)$ extracted from $\alpha_{s}\left(E_{T}\right)$ is shown in the inset along with the weighted average as the horizontal line. shown in the inset of Fig. 1. Averaging over the range 40-250 GeV, we obtain

$$
\alpha_{s}\left(M_{Z}\right)=0.1178 \pm 0.0001 \text { (stat). }
$$

Inclusion of the data with $E_{T}>250 \mathrm{GeV}$ results in an increase of the average value by 0.0001 .

The running of $\alpha_{s}$ is tested by verifying if $\alpha_{s}\left(M_{Z}\right)$ is independent of the energy scale $E_{T}$ at which the jet cross section is measured. The 27 values of $\alpha_{s}\left(M_{Z}\right)$ obtained from the data in the jet $E_{T}$ range $40-250 \mathrm{GeV}$ are fit to the linear function $P_{0}+P_{1} \times E_{T}$. The fit yields $P_{0}=$ $0.1173 \pm 0.0005$ and $P_{1}=(0.3 \pm 0.3) \times 10^{-5} \mathrm{GeV}^{-1}$ with $\chi^{2} /$ d.o.f. $=1.3$, showing that $\alpha_{s}\left(M_{Z}\right)$ is independent of $E_{T}$ within one standard deviation. The estimated experimental systematic uncertainty on the value of $P_{1}$ is $\pm 5.0 \times 10^{-5} \mathrm{GeV}^{-1}$. The nontrivial result of this fit demonstrates the correctness of the QCD prediction for the evolution of $\alpha_{s}$ over the above range.

The experimental systematic uncertainties on the value of $\alpha_{s}\left(M_{Z}\right)$ are derived from those on the inclusive jet cross section. For each source of systematic uncertainty described below, except normalization, the inclusive jet cross section was reevaluated by varying the corresponding parameter in the detector response by $1 \sigma$. For the normalization uncertainty, it was changed by a scale factor [13]. These uncertainties were propagated to $\alpha_{s}\left(M_{Z}\right)$ by repeating the procedure described above using the spectra given in Table VI of Ref. [13]. The results are shown in Fig. 2. The deviations of $\alpha_{s}\left(M_{Z}\right)$ for each spectrum from the central value are given in Table I. The dominant experimental systematic uncertainty in the inclusive jet cross section measurement is due to the calorimeter response to jets.

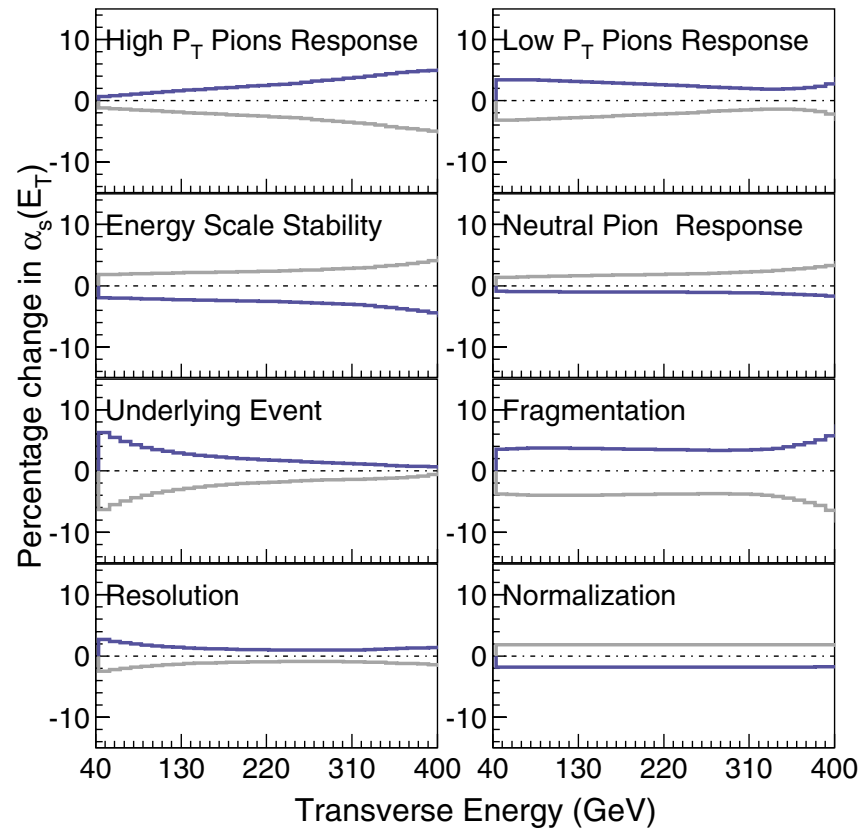

FIG. 2. Experimental systematic uncertainties for $\alpha_{s}$ measurement (the lines are 1 standard deviation contours), with CTEQ4M as input PDF and $\mu_{R}=\mu_{F}=E_{T}$. 
TABLE I. Experimental systematic uncertainties on $\alpha_{s}\left(M_{Z}\right)$ extracted using CTEQ4M parton distribution functions.

\begin{tabular}{lcc}
\hline \multicolumn{1}{c}{ Source of uncertainty } & $\Delta \alpha_{s}$ & $\left(\frac{\Delta \alpha_{s}}{\alpha_{s}}\right) \%$ \\
\hline Calorimeter high $P_{T}$ pion response & +0.0036 & +3.1 \\
Calorimeter low $P_{T}$ pion response & -0.0055 & -4.7 \\
& +0.0027 & +2.3 \\
Energy scale stability & -0.0033 & -2.8 \\
& +0.0030 & +2.6 \\
Neutral pion response & -0.0030 & -2.6 \\
Underlying event energy & +0.0016 & +1.4 \\
& -0.0021 & -1.8 \\
Jet fragmentation functions & +0.0025 & +2.1 \\
& -0.0027 & -2.3 \\
Jet energy resolution & +0.0046 & +3.9 \\
Normalization & -0.0044 & -3.7 \\
& +0.0015 & +1.3 \\
\hline \hline
\end{tabular}

The detector response and jet energy corrections are derived from a combination of test-beam data and Monte Carlo simulations. The calorimeter response to charged pions was evaluated separately for high and low transverse momentum $\left(P_{T}\right)$ pions from test-beam data and isolated charged tracks from $p \bar{p}$ data with an uncertainty of $\pm 5 \%$ for $P_{T} \leq 5 \mathrm{GeV}, \pm 3 \%$ for $5 \mathrm{GeV}<P_{T}<15 \mathrm{GeV}$ and ${ }_{-2.0}^{+3.6} \%$ for $P_{T} \geq 15 \mathrm{GeV}$. During the run, the calorimeter response was monitored by using muons, isolated particles, and the measured inclusive jet cross section. The response was found to be stable within $\pm 1 \%$. The electromagnetic calorimeter was calibrated using electrons from $p \bar{p}$ interactions. The associated uncertainty, labeled in Fig. 2 as neutral pion response, arises from the modeling of calorimeter response to very low energy electrons. The underlying event energy (nonjet energy contribution to the jet $E_{T}$ ) was measured from minimum bias data, and its mean value was varied by $\pm 30 \%$ [13] to evaluate the effect on the jet cross section. The error from the jet fragmentation functions is due to the extrapolation of the track momentum and multiplicity distribution to the high $E_{T}$ region and from uncertainties in the track reconstruction efficiency. The detector jet energy response has a Gaussian shape with exponential tails on both the high and low sides and a resolution with an uncertainty of $\pm 10 \%$. Finally, the overall normalization of the inclusive jet cross section has an uncertainty of $\pm 4.5 \%$, dominated by the contribution from the total cross section measurement. Summing in quadrature all the above uncertainties after propagation to $\alpha_{s}\left(M_{Z}\right)$ yields a total experimental systematic uncertainty of $\pm_{0.0095}^{0.0081}$.

The theoretical uncertainties are mainly due to the choice of renormalization and factorization scales and parton distribution functions. The scales $\mu_{F}$ and $\mu_{R}$ are expected to be of the same order as the characteristic scale of the process, which in this case is the jet $E_{T}$. We have evaluated the changes in $\alpha_{s}\left(M_{Z}\right)$ resulting from independently varying $\mu_{F}$ and $\mu_{R}$ from $E_{T} / 2$ to $2 E_{T}$ and found that the largest changes occur for $\mu_{R}=\mu_{F}$. For all results presented in this Letter the two scales were set equal. The sensitivity of the measured $\alpha_{s}\left(M_{Z}\right)$ to changes in these scales is indicated by the shaded band in Fig. 3(a). Over the $E_{T}$ range from 40 to $250 \mathrm{GeV}$, the shift in $\alpha_{s}\left(M_{Z}\right)$ induced by changing the scale from $E_{T} / 2\left[\alpha_{s}\left(M_{Z}\right)=0.1129 \pm 0.0001\right]$ [23] to $2 E_{T}\left[\alpha_{s}\left(M_{Z}\right)=0.1249 \pm 0.0001\right]$ is approximately ${ }_{-4 \%}^{+6 \%}$, independently of $E_{T}$.

The coefficients $\hat{X}_{0}$ and $k_{1}$ in Eq. (1) depend on the PDFs, which are obtained from global fits to deep inelastic scattering (DIS), Drell-Yan production, and other collider data, including the inclusive jet results from Tevatron. Each PDF set has an associated strong coupling constant, $\alpha_{s}^{\mathrm{PDF}}$. The gluon PDF $[G(x)]$ determined from the fit is highly correlated with $\alpha_{s}^{\mathrm{PDF}}$ because, in equations describing all the processes used, the $G(x)$ is always accompanied by $\alpha_{s}^{\mathrm{PDF}}$. To calculate the above coefficients, the PDFs are evolved using $\alpha_{s}^{\mathrm{PDF}}$. For this procedure of measuring $\alpha_{s}$ to be valid, the extracted value of $\alpha_{s}$ should be consistent with the input $\alpha_{s}^{\mathrm{PDF}}$, although not necessarily equal. The variation in parton distributions, especially in the gluon distribution, allowed by the world data was studied by the CTEQ Collaboration by fixing the value of $\alpha_{s}^{\mathrm{PDF}}$ to $0.110,0.113,0.116,0.119$, or 0.122 , with resulting $\chi^{2}$ of $1388,1323,1323,1388$, or 1543 for 1297 nonjet data points [22]. We use the CTEQ4A series to study the $\alpha_{s}\left(M_{Z}\right)$ dependence on the PDFs. In addition, we have studied $\alpha_{s}\left(M_{Z}\right)$ using PDF sets which do not include Tevatron jet results, such as the $\operatorname{MRST}(\mathrm{g} \uparrow)$ set [24], the MRSA' series [25], and two MRS-R sets [26]. The $\chi^{2}$, calculated by comparing the data with the theoretical prediction in

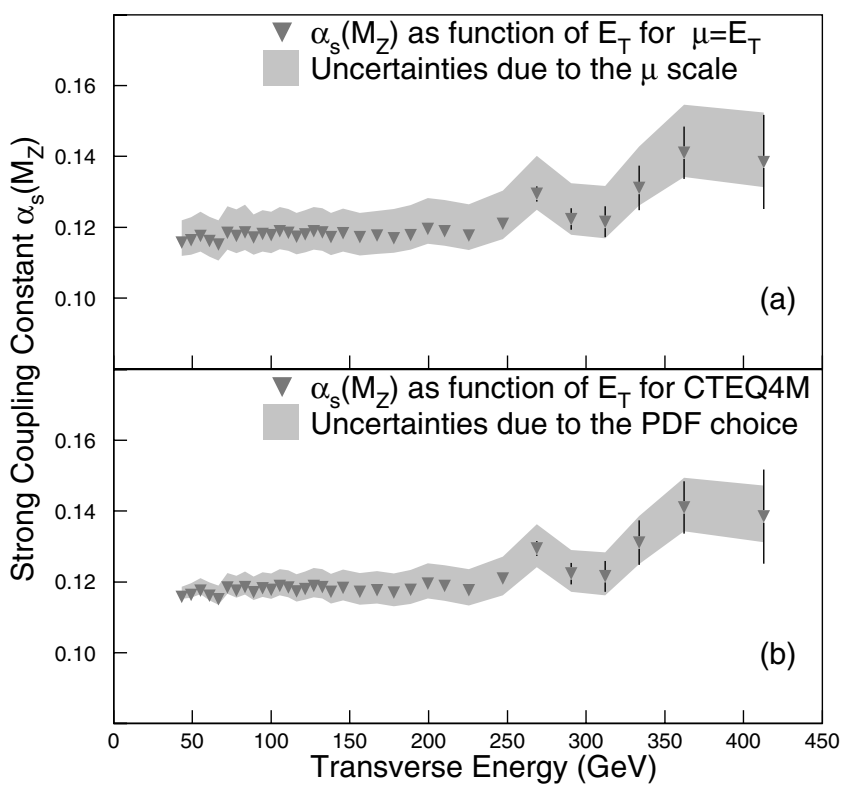

FIG. 3. Uncertainties in $\alpha_{s}\left(M_{Z}\right)$ due to the renormalization scale $\mu,(\mathrm{a})$, and parton distribution functions, (b). 
the restricted range of $40-250 \mathrm{GeV}$, is used to quantify the agreement. The minimal $\chi^{2} /$ d.o.f. $=1.38$ is obtained for CTEQ4M $\left(\alpha_{s}^{\mathrm{PDF}}=0.116\right)$, therefore we use this PDF in our final fit. Excluding the PDFs which have obvious disagreement $\left(\chi^{2} /\right.$ d.o.f. $\left.\geq 5\right)$, we estimate the uncertainty on the $\alpha_{s}\left(M_{Z}\right)$ due to the PDF choice to be $\pm 5 \%$.

Finally, the variation of $\mathcal{R}_{\text {sep }}$, the jet clustering parameter, from 1.3 to 2.0 results in a $5 \%-7 \%$ normalization change of the inclusive jet cross section. The corresponding variation in the $\alpha_{s}\left(M_{Z}\right)$ measurement is $2 \%-3 \%$.

To explore the flexibility in the gluon distribution at high $E_{T}$, a special PDF set, CTEQ4HJ, was generated by including CDF jet data in the global fit with higher statistical weight assigned to high $E_{T}$ points and a new parametrization of the gluon distribution [22]. This PDF yields good agreement between Tevatron data and theoretical predictions. Using this set, we obtain $\alpha_{s}\left(M_{Z}\right)=0.1185 \pm$ 0.0001 , averaged over the entire $E_{T}$ range.

In conclusion, we have tested the evolution of the strong coupling constant $\alpha_{s}$ using the inclusive jet cross section data from $\bar{p} p$ collisions at $\sqrt{s}=1800 \mathrm{GeV}$. Our results demonstrate that for $E_{T}$ in the range of $40-250 \mathrm{GeV}$ with $\mu_{R}=E_{T}$ the running of $\alpha_{s}$ is in good agreement with QCD predictions. The value of $\alpha_{s}$ expressed at the $Z^{0}$ boson mass is found to be

$$
\alpha_{s}\left(M_{Z}\right)=0.1178 \pm 0.0001 \text { (stat) }{ }_{-0.0095}^{+0.0081} \text { (expt. syst) } .
$$

This value is in good agreement with the world average $\alpha_{s}\left(M_{Z}\right)=0.1181 \pm 0.0020$ [27]. The theoretical uncertainties associated with the choice of parton distribution functions $(\sim 5 \%)$ and the choice of the renormalization scale $\left({ }_{-4 \%}^{+6 \%}\right)$ are comparable to the experimental systematic uncertainty.

We thank W. Giele for providing the JETRAD program and for helpful discussions and comments on all stages of this work. We thank the Fermilab staff and the technical staffs of the participating institutions for their vital contributions. This work was supported by the U.S. Department of Energy and National Science Foundation; the Italian Istituto Nazionale di Fisica Nucleare; the Ministry of Education, Science, Sports and Culture of Japan; the Natural Sciences and Engineering Research Council of Canada; the National Science Council of the Republic of China; the Swiss National Science Foundation; the A.P. Sloan Foundation; the Bundesministerium fuer Bildung und Forschung, Germany; the Korea Science and Engineering Foundation (KoSEF); the Korea Research Foundation; and the Comision Interministerial de Ciencia y Tecnologia, Spain.
*Now at Northwestern University, Evanston, Illinois 60208.

${ }^{\dagger}$ Now at University of California, Santa Barbara, California 93106.

${ }^{\ddagger}$ Now at Carnegie Mellon University, Pittsburgh, PA 15213.

[1] H. Fritzsch, M. Gell-Mann, and H. Leutwyler, Phys. Lett. 47B, 365 (1973).

[2] G. 't Hooft (unpublished); D. Gross and F. Wilczek, Phys. Rev. Lett. 30, 1343 (1973); H. D. Politzer, Phys. Rev. Lett. 30, 1346 (1973).

[3] P. Abreu et al., Phys. Lett. B 398, 194 (1997).

[4] P. Abreu et al., Eur. Phys. J. C 14, 557 (2000).

[5] P. Pfeifenschneider et al., Eur. Phys. J. C 17, 19 (2000).

[6] T. Coan et al., Phys. Lett. B 356, 580 (1995); R. Barate et al., Eur. Phys. J. C 4, 409 (1998); K. Ackerstaff et al., Eur. Phys. J. C 7, 571 (1999).

[7] J. Santiago and F. J. Yndurain, Nucl. Phys. (Proc. Suppl.) 86, 69 (2000); C. Adloff et al., Eur. Phys. J. C 21, 33 (2001).

[8] J. Breitweg et al., Phys. Lett. B 507, 70 (2001); C. Adloff et al., Eur. Phys. J. C 19, 269 (2001).

[9] J. H. Kim et al., Phys. Rev. Lett. 81, 3595 (1998).

[10] D. Abbaneo et al., CERN-EP/2001-021 (2001).

[11] I. Hinchliffe and A. V. Manohar, Annu. Rev. Nucl. Part. Sci. 50, 643 (2000).

[12] F. Abe et al., Nucl. Instrum. Methods Phys. Res., Sect. A 271, 387 (1988).

[13] T. Affolder et al., Phys. Rev. D 64, 032001 (2001).

[14] W. Giele, E. W. N. Glover, and J. Yu, Phys. Rev. D 53, 120 (1996).

[15] S. D. Ellis, Z. Kunszt, and D. E. Soper, Phys. Rev. Lett. 69, 3615 (1992).

[16] W. Giele, E. W. N. Glover, and D. A. Kosower, Phys. Rev. Lett. 73, 2019 (1994).

[17] W. Giele, E. W. N. Glover, and D. A. Kosower, Nucl. Phys. B403, 633 (1993).

[18] R. K. Ellis and J. Sexton, Nucl. Phys. B269, 445 (1986).

[19] S. D. Ellis, Z. Kunszt, and D. E. Soper, Phys. Rev. Lett. 64, 2121 (1990).

[20] F. Aversa et al., Phys. Lett. B 211, 465 (1988).

[21] W. A. Bardeen et al., Phys. Rev. D 18, 3998 (1978).

[22] H. L. Lai et al., Phys. Rev. D 55, 1280 (1997).

[23] The $\alpha_{S}\left(M_{Z}\right)$ value quoted in the preprint by Mesropian [FERMILAB-CONF-99-177-E (1999)] was obtained with $\mu_{R}=E_{T} / 2$; however, for this Letter we repeated the analysis with $\mu_{R}=E_{T}$.

[24] A. D. Martin et al., Eur. Phys. J. C 4, 463 (1998).

[25] A. D. Martin, R. Roberts, and W. J. Stirling, Phys. Lett. B 356, 89 (1995).

[26] A. D. Martin, R. Roberts, and W. J. Stirling, Phys. Lett. B 387, 419 (1996).

[27] Particle Data Group, D. E. Groom et al., Eur. Phys. J. C 15, 1 (2000). 